\title{
MUSCULOSKELETAL
}

\section{Ultrasound elastography findings in piriformis muscle syndrome}

\author{
Adnan Demirel, Murat Baykara1', Tuba Tülay Koca, Ejder Berk \\ Departments of Physical Medicine and Rehabilitation and 'Radiology, Faculty of Medicine, Sütçü İmam University, \\ Kahramanmaraş, Turkey
}

Correspondence: Asst. Prof. Tuba Tülay Koca, Departments of Physical Medicine and Rehabilitation, Faculty of Medicine, Sütçü Imam University, Kahramanmaraş, Turkey. E-mail: tubatulaykoca@outlook.com

\begin{abstract}
Background: Piriformis muscle syndrome (PMS) is relatively less known and underestimated because it is confused with other clinical pathological conditions. Delays in its diagnosis may lead to chronic somatic dysfunction and muscle weakness. Objective: Here, we aimed to evaluate the diagnostic performance of the ultrasound elastography (UE) as an easy, less-invasive, and cost-effective method for early diagnosis of PMS. Materials and Methods: Twenty-eight cases clinically diagnosed as PMS at the outpatient clinic were evaluated by UE. The elastographic strain ratio was calculated by dividing the strain value of the subcutaneous fat tissue by the mean stress value of the muscle beneath. The diagnostic performances of the strain rate measures were compared using the receiver operating characteristic curve analysis. Results: Twenty-one $(N=21)$ cases were female, and seven $(N=7)$ of the cases were male. The mean age was 45 years (ranged 24-62 years). The strain rates of piriformis muscle (PM) and gluteus maximus (GM) muscles were significantly higher on the PMS-diagnosed side $(P<0.001)$. The cutoff value of UE strain ratio for the PM and GM were 0.878 [95\% confidence interval $(\mathrm{Cl}) 0.774-0.981$ ] and $0.768(95 \% \mathrm{Cl} 0.622-0.913)$, respectively, and the sensitivity and specificity values were, respectively, $80.95 \%$ and $85.71 \%$ for the PM, and they were, respectively, $85.71 \%$ and $66.67 \%$ for the GM. Conclusion: We showed that the muscle elasticity and tissue hardening increased on the problematic side both on PM and GM. UE may provide early diagnosis of PMS, thereby increasing the possibility of treatment with less invasive methods.
\end{abstract}

Key words: Elastography; piriformis muscle syndrome; ultrasound

\section{Introduction}

Piriformis muscle syndrome (PMS) is a neuropathy that occurs due to the compression or irritation of the sciatic nerve by the piriformis muscle (PM). It accounts for $6-8 \%$ of the back and sciatic pain cases. This clinical condition is relatively less known and underestimated because it is confused with other clinical pathological conditions. Delays in its diagnosis may lead to chronic somatic dysfunction and muscle weakness due to compensatory changes..$^{[1-3]}$ PMS is diagnosed by history, physical examination, and radiological evaluations which are used for differential

\begin{tabular}{|l|l|}
\hline \multicolumn{2}{|c|}{ Access this article online } \\
\hline Quick Response Code: & \\
\hline & Website: \\
\hline & www.ijri.org \\
& DOI: \\
& $10.4103 /$ /ijri.IJRI_133_18 \\
\hline
\end{tabular}

diagnosis. Up to date, there is no gold standard diagnostic method in PMS.

The development of ultrasound technology resulted in the emergence of ultrasound elastography (UE) that can directly measure the mechanical properties of tissue, including muscle stiffness. Its real-time and direct measurements of muscle stiffness can aid the diagnosis of musculoskeletal injuries. It can also help monitor outcomes of interventions affecting muscle in neuromuscular and musculoskeletal diseases, and it can better inform the functional prognosis.

This is an open access journal, and articles are distributed under the terms of the Creative Commons Attribution-NonCommercial-ShareAlike 4.0 License, which allows others to remix, tweak, and build upon the work non-commercially, as long as appropriate credit is given and the new creations are licensed under the identical terms.

For reprints contact: reprints@medknow.com

Cite this article as: Demirel A, Baykara M, Koca TT, Berk E. Ultrasound elastography findings in piriformis muscle syndrome. Indian J Radiol Imaging 2018;28:412-8. 
But more information is needed on the use and limitations for correct and effective application of the technique. ${ }^{[4-6]}$

Here, first in the literature, we aimed to evaluate the diagnostic performance of UE as an easy, less-invasive, and cost-effective method for early diagnosis of PMS.

\section{Materials and Methods}

\section{Cases}

A detailed physical examination was performed in the outpatients' clinic for the patients with the complaints of increased hip pain during sitting, squatting, or standing up, and of numbness and weakness in the legs. The patients with sensitivity in the sacroiliac joint, the greater sciatic notch, the PM, and the regions surrounding the PM; palpated mass in ipsilateral hips; moderate relaxation in pain by traction of the affected hip; asymmetric weakness in the affected hip; positive results of Laseque and Beatty tests (+); positive results of Freiberg, and Pace tests $(+)$ on the painful side; and the finding of limited internal rotation in the lower extremity were diagnosed as PMS clinically. ${ }^{77,8]}$ The patient has an antalgic gait during walking with the positive results of Lasègue, Pace, and Freiberg tests that are diagnosed as PMS. ${ }^{[9]}$ The sensitivity localized in the PM during $90^{\circ}$ flexure of the hip and extension of the knee indicates the presence of the Lasègue symptom. The positive sign for pace test (FAIR test) is the pain formation when the hip is forced to internal rotation and adduction by stabilizing the pelvis while the patient's painful side is upside down, and the hip and knee are in the flexion position. ${ }^{[9,10]}$ The Freiberg symptom is the formation of pain in the PM when a resistant external rotation is forced for the leg that has been passively internally rotated. ${ }^{[11]}$

The patients with any other pathological condition of the sciatic nerve were not included in the study. The results of the patients' magnetic resonance imaging (MRI) of lumbar or hips, performed within the last year, were used to exclude differential diagnosis. Those with history of gluteal injection were not included in the study. Intramuscular injections may cause changes in muscle structure depending on the type of drug, the solubility in water or in the oil, and the duration of its effect. These also affect muscle elasticity results. Patients were questioned whether there had any pelvic or abdominal pathologies. Then, the patients who were diagnosed as PMS by physiatrist at outpatient clinic were referred to the study-blind radiologist.

\section{Ultrasound elastography}

UE technique is a new functional US imaging technique developed in the last 5 years that can demonstrate the distribution of tissue elasticity. The low strain ratio indicated the decrease in tissue elasticity, softening of the tissue, loss of tendon integrity, and decrease in quality. Unlike other methods, this technique provided dynamic data. ${ }^{[12]}$
A high-resolution ultrasonography system (AplioTM 400 Platinum, Toshiba Medical Systems Corporation, Tochigi, Japan) and a broadband convex probe (PVT-375BT) were used by a same trained radiologist. The gray-scale ultrasonographic posterior images of the gluteal region were obtained symmetrically, bilaterally, and separately. The patient was lying on a flat surface in the prone position during the application. The elastography mode was activated, and pressure was applied when the gluteus maximus muscle (GM) and PM were captured in the same image during compression phase [Figure 1]. The UE strain value (UESV) of the subcutaneous fat tissue in the same image and UESV of the central segments of the PM and GM were detected using the region of interest (ROI). The UE strain ratio (UESR) of the muscles was calculated by dividing the fat values by the muscle values. All procedures were performed by the same experienced radiologist who was kept uninformed about the clinical diagnosis.

\section{Statistical assessment}

The statistical evaluation was conducted using the SPSS version 21 (SPSS Inc., IL, USA). The KolmogorovSmirnov test was applied to check the distribution of parameters. Categorical data were presented as numbers and percentages. The independent Student $t$-test was used to determine the difference in normally distributed data, and the Mann-Whitney $U$ test was used for comparing medians for the non-parametrically distributed variables. Spearman's rho method was used for assessing correlations between the strain rates and for the comparisons, whereas the diagnostic performances were compared with receiver operating characteristic (ROC) curve analysis.

The study's ethic committee approval was taken from local center (06.15.2016/protocol number is 146) and is complied with Helsinki Declaration principles. Informed consent forms were obtained from the patients.

\section{Results}

Twenty-one $(N=21)$ cases were female and seven $(N=7)$ of the cases were male. Their ages ranged from 24 to 62 years, and the median age was 45 years [Table 1]. The UE results showed a significant difference in elasticity of the PMs between the painless and painful sides [Figure 1]. Right-side dominance $(62.5 \%$ in females, $100 \%$ in males, and $71.4 \%$ in total) was observed.

The mean UESR values of the muscles on the side clinically diagnosed with PMS were as follows: PM, $1.59 \pm 1.21$ and its median ( $\mathrm{min} / \mathrm{max}), 1.30(0.55 / 4.86) ; \mathrm{GM}, 1.01 \pm 1.05$ and its median $(\mathrm{min} / \mathrm{max}), 0.68(0.25 / 4.19)$. The mean UESR values on the normal side were as follows: $\mathrm{PM}, 0.5 \pm 0.47$ and its median (min/max), 0.57 (0.02/1.71); GM, $0.42 \pm 0.35$ and its median ( $\mathrm{min} / \mathrm{max}), 0.36(0.02 / 1.17)$. The UESR values of PM and GM were significantly higher on the painful 

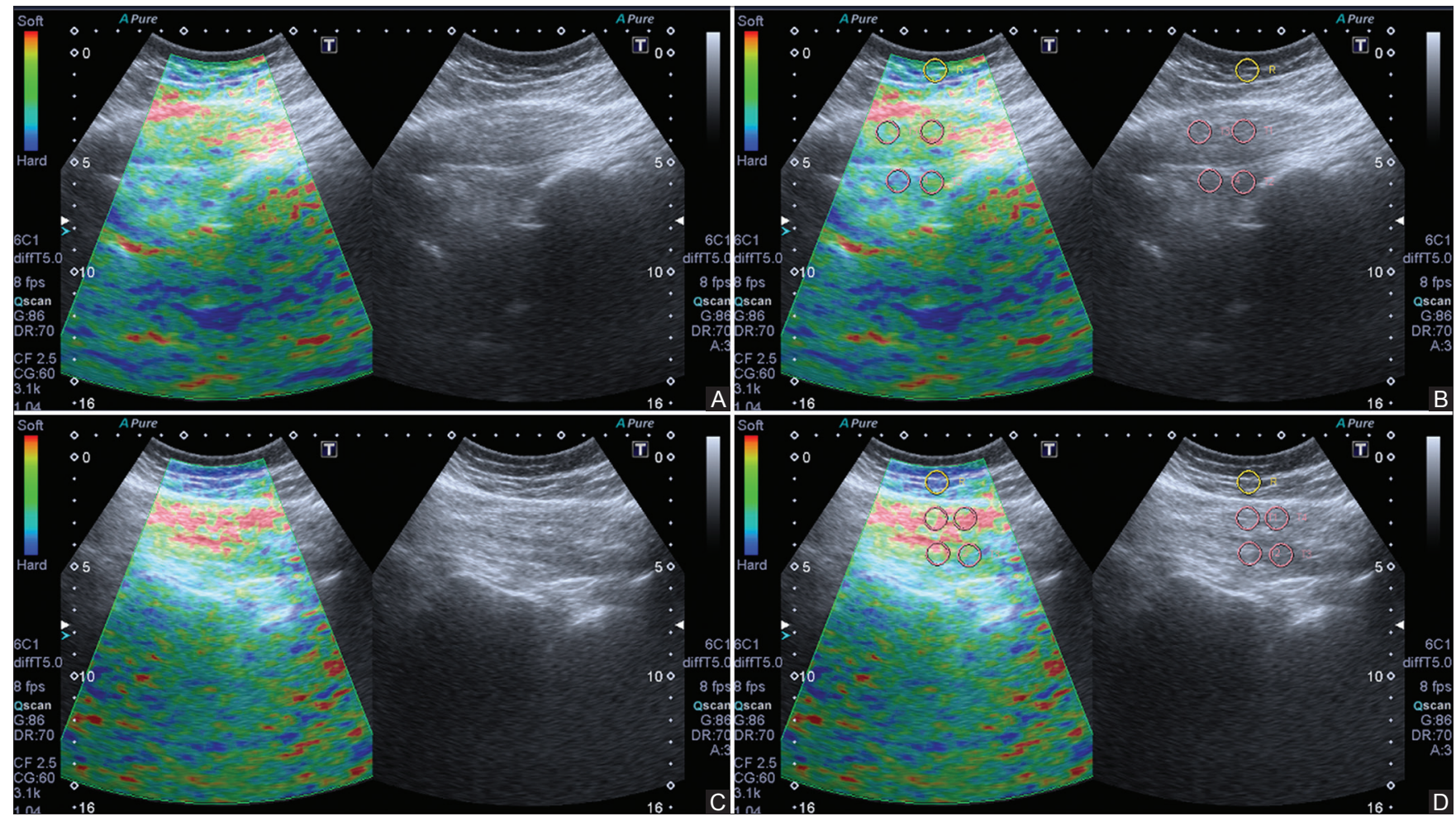

Figure 1 (A-D): Ultrasonographic elastography images from a 24-year-old female patient. ( $A$ and B) Right and $(C$ and $D)$ left gluteal regions

Table 1: Descriptive and analytic characteristics of the study

\begin{tabular}{|c|c|c|c|}
\hline Age, min-max/median (years) & \multicolumn{3}{|c|}{$24-62 / 45$} \\
\hline Gender (female/male) & \multicolumn{3}{|c|}{$21 / 7$} \\
\hline Right-side dominance (\%) & \multicolumn{3}{|c|}{$\begin{array}{c}62.5 \% \text { in females, } 100 \% \text { in males, and } 71.4 \% \\
\text { in total }\end{array}$} \\
\hline & PMS side & Normal side & $P$ \\
\hline \multicolumn{4}{|l|}{ UESR, PM* } \\
\hline Mean \pm SD & $1.59 \pm 1.21$ & $0.5 \pm 0.47$ & 0.00 \\
\hline Median/Minimum-maximum & $1.30(0.55 / 4.86)$ & $0.57(0.02 / 1.71)$ & \\
\hline \multicolumn{4}{|l|}{ UESR, GMs* } \\
\hline Mean \pm SD & $1.01 \pm 1.05$ & $0.42 \pm 0.35$ & 0.003 \\
\hline Median/Minimum-maximum & $0.68(0.25 / 4.19)$ & $0.36(0.02 / 1.17)$ & \\
\hline
\end{tabular}

*Statistically significant difference, $P<0.05$. UESR: Ultrasound elastography strain ratio; PM: Piriformis muscle; GMs: Gluteus maximus; PMS: Piriformis muscle syndrome; SD: Standard deviation

side compared with the normal side $(P=0.000$ and 0.003 , respectively) [Table 1 and Figure 2A-D].

For UESRs, the values under the ROC curve were 0.878 [95\% confidence interval (CI) 0.774-0.981] and 0.768 (95\% CI 0.622-0.913) for PM and GM, respectively. When UESR values 0.765 and 0.420 were used as cutoff for the PM and $\mathrm{GM}$, respectively, the sensitivity and specificity values were, respectively, $80.95 \%$ and $85.71 \%$ for the $\mathrm{PM}$, and they were, respectively, $85.71 \%$ and $66.67 \%$ for the GM [Figure 3].

When $124.62 \%$ increase in UESR relative to the normal side was applied on the PM, the sensitivity and specificity values were both $100 \%$. When $100.04 \%$ increase in UESR was applied on the GM, the sensitivity and specificity values were $100 \%$ and $95.24 \%$, respectively. It is shown that the PM UESR/GM UESR increases with age, and the increase in UESR of both PM and GM with height was higher on the right side. The increase in the PM UESR/GM UESR with visual analog scale (VAS) and the increase in the PM UESR/GM UESR with weight were higher on the left side.

\section{Correlations}

A correlation was observed between the UESR values of the PM (rho = 1.000; $P=0.000)$ and the GM (rho = 1.000; $P=0.000)$ on the affected side, and for all PM and GM UESR values (rho $=1.000, P=0.000)$. Also, the disease was observed at higher incidences on the right side $(\mathrm{rho}=-0.788$, $P=0.000,62.5 \%$ in females, $100 \%$ in males, and $71.4 \%$ in total). With age, the UESR values of the GM increased not only on the left $(\mathrm{rho}=0.607 ; P=0.001)$ and right $(\mathrm{rho}=0.632$, $P=0.002)$ side together but also on the normal ( $\mathrm{rho}=0.572$, $P=0.007)$ and pathologic $($ rho $=0.655, P=0.002)$ sides. The prevalence of high PM UESR/GM UESR decreases with age (rho $=-0.743 ; P=0.000$ ).

As the UESR values of the muscle (rho $=0.667, P=0.001)$ and VAS (rho $=0.621 ; P=0.003$ ) increased, the positive results from the Laseque test also increased whereas findings of mass palpation $(\mathrm{rho}=0.442 ; P=0.045)$ decreased.

\section{Discussion}

PM is a flat pyramidal-shaped muscle..$^{[1]}$ The anterior side of the PM originates around the sacroiliac joint capsule close 

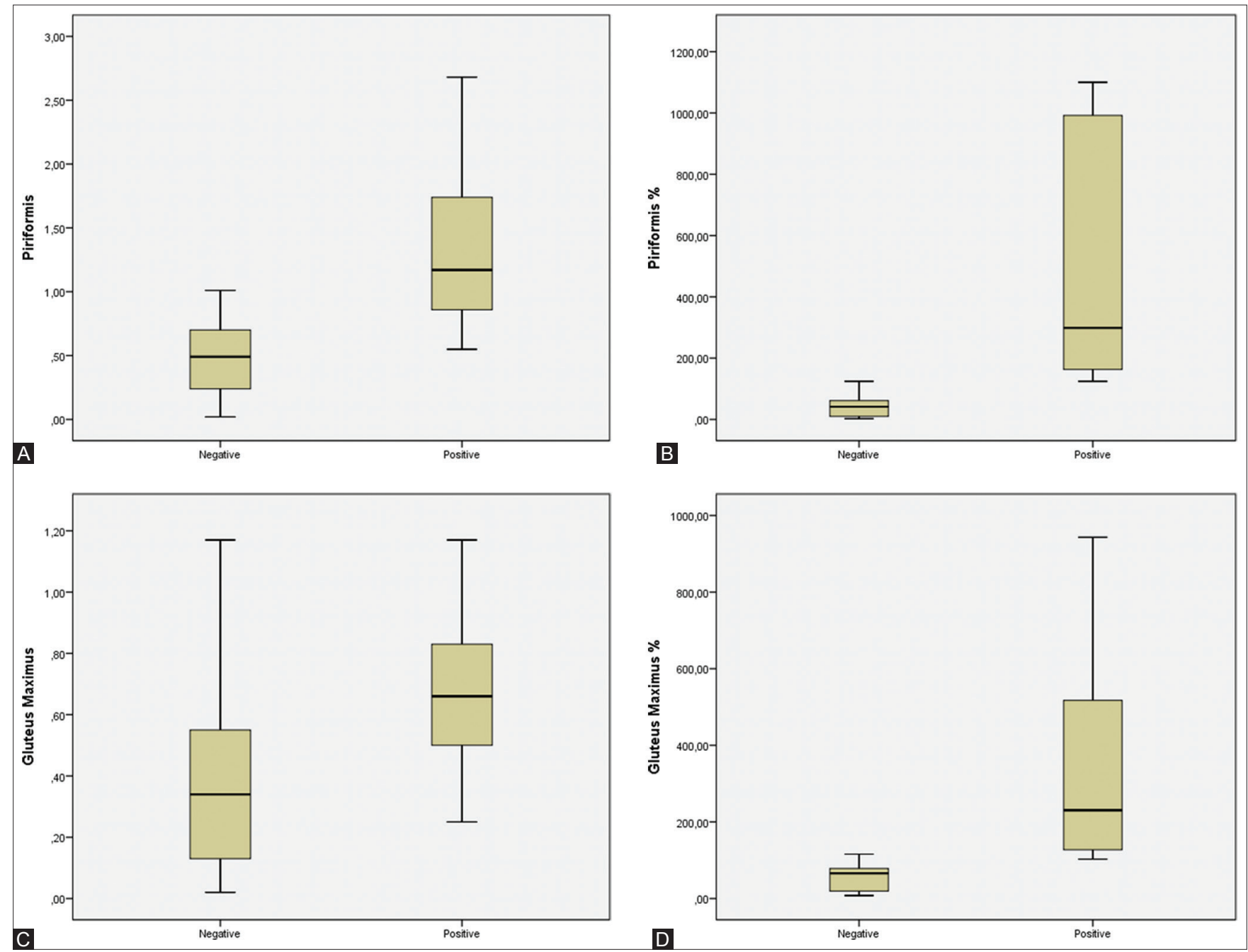

Figure 2 (A-D): Ultrasound elastography strain ratio values and percentage (\%) change rates of piriformis muscles (A and B) and gluteus maximus muscles $(C$ and $D)$ according to piriformis muscle syndrome existence were shown at the graph

to the S2-S4 level, passes through the large sciatic foramen, and attaches to the upper surface of the greater trochanter of the femur. It is adjacent to the obturator internus and the gemellus muscles. ${ }^{[2,3]}$ The function of PM depends on the position of the hip. PM is innervated by the spinal roots, S1 and S2, and rarely by L5. The PM is responsible for the external rotation of the hip when the hip is in neutral position and for the abduction of the hip when the hip is in the flexion. It provides postural stability while standing and walking. ${ }^{[4]}$ Yeoman described the syndrome for the first time in 1928, and Robinson created a six-item diagnostic list for it in $1947 .{ }^{[13,14]}$ PMS may result from the variability in the anatomical relationship between the PM and the sciatic nerve, or it could occur secondary to the micro- or macrotraumas of the PM. ${ }^{[15]}$

The incidence of PMS increases in the fourth and fifth decades, and its clinical diagnosis is difficult. The probable reason why females suffer from PMS six times more frequently than males is that the quadriceps femoris muscle biomechanically has a wider angle in the female pelvis than in the male pelvis. The actual number of the cases is expected to be higher than the diagnosed ones because PMS is confused with other clinical pathologic conditions. Therefore, realistic epidemiological information is not available. ${ }^{[15-17]}$ We found similar results coherent with the literature about data of age, gender, and prevalence. The fact that female gender has near-bilateral values, the increase in the weight load on the right leg with age and increased use of the right leg in males (a right-handed society) suggest that these factors affect the variability in the values.

The delayed diagnosis of this is not well known and not well considered. It can lead to pain, paresthesia, hyperesthesia, and muscle weakness caused by the compression of the sciatic nerve, chronic somatic dysfunction, and compensatory changes. This makes the treatment more difficult and invasive ${ }^{[18]} \mathrm{A}$ differential diagnosis should be considered for patients in pain, especially for the females. The conditions causing lower back pain and sciatic pain such as sacroiliac joint dysfunction, facet syndrome, spinal stenosis, trochanteric bursitis, myofascial pain syndrome, 


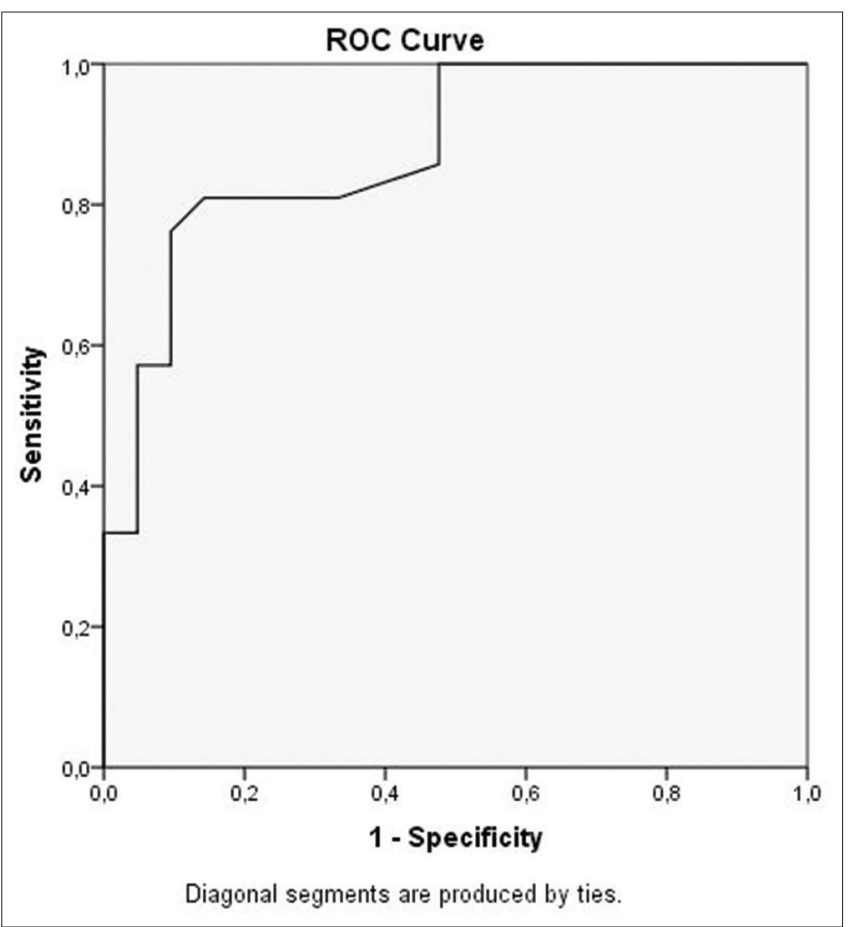

Figure 3: Receiver operating characteristic analysis for ultrasound elastography strain ratio values of PM and GM strain ratios and percent changes

pelvic tumors, endometriosis, and the conditions causing irritation of the sciatic nerve should be considered for the differential diagnosis of PMS. ${ }^{[19,20]}$

MRI and electrophysiological evaluation can be used for the differential diagnosis although they are not the definitive diagnostic tests. However, imaging modalities, such as MRI, have become a critical component in assisting with the diagnosis; many anatomic variants may have gone undiagnosed in the past due to the lack of or limitations of imaging studies. ${ }^{[21]}$ An advanced technique of MR neurography may also be useful in aiding the diagnosis of this condition. ${ }^{[22]}$ While not addressing variant sciatic nerve anatomy on imaging and associated clinical findings, prior studies have suggested a relationship between MRI findings and the clinical diagnosis of piriformis syndrome in the form of asymmetric muscle bulk detectable on routine MRI or ultrasound (with increased piriformis size on the affected side) and increased fluid signal in the sciatic nerve at the sciatic notch on MR neurography. ${ }^{[23]}$ Huang et al. found that the thickness, area, and volume of the pathological side of PM in the PMS group were all significantly higher than normal side in MRI-based study. ${ }^{[24]}$

The diagnostic difficulties can be overcome by using the UE method, which is increasingly used in the recent years. UE is an ultrasound-based technique that determines mechanical properties of the tissue quantitatively, visually, and qualitatively compared with the B-mode analysis (acoustic impedance) or the color Doppler ultrasound (vascular blood flow). Because the stiffness of the tissue is determined by this technique, obtaining a diagnosis by comparing the elasticity (and stiffness) of the PM is possible. Strain elastography is the most commonly used measurement technique among several others, and the externally applied pressure causes more deformation in the soft tissues and less deformation in the hard tissues. This information is color-coded on a screen, and the quantitative data are obtained by measurements from the ROI. ${ }^{[12,25,26]}$

Indeed, MRI can be a functional method for a correct PMS diagnosis by evaluating lumbar hernia, lumbar stenosis, and sacroiliac syndromes that cause sciatic nerve symptom, and other differential diagnoses such as pathologies located around the PM. Depending on the etiology, MRI with fluid-sensitive sequences may visualize the increased nerve intensities at the level of the greater sciatic foramen, the increased intensity of the PM, the muscular hypertrophy, or the sciatic nerve compressed by an accessory PM fiber compared with the asymptomatic side. . $33,27,28]^{2}$

This novel study demonstrated that PMS could be quantitatively diagnosed using the UE method. Despite being a relatively new method, the clinical use of UE has rapidly increased. This method used to evaluate the elasticity of the tissues takes the tissue composition as a reference. ${ }^{[4,26,27]}$ It would not be wrong to say that in muscular pathologies, the elasticity of the muscles changes according to the extent of their own contraction without a change in their tissue composition compared with other tissues. Reflexively increasing contraction results in reduced elasticity in pathological conditions. ${ }^{[22,27,28]}$ The present study showed that the muscle elasticity increased on the problematic side. Evaluating with the PM indicated that the GM was also affected on the problematic side and that relative comparison of both muscles could provide more reliable information with $79 \%$ of success rate. ${ }^{[8,29]}$

Anti-inflammatory drugs and muscle relaxants can be used for the treatment. During acute period, cold application and stretching exercises are applied to $\mathrm{PM}$, hip abductor, and adductor muscles. ${ }^{[29-31]}$ In subacute and chronic periods, superficial and deep heaters are applied among other physiotherapy modalities. Intramuscular injections of local anesthetic or corticosteroids can be given for treating PMS. Intramuscular injections of botulinum toxin (Botox) is an effective approach for cases where an adequate response has not been achieved. ${ }^{[8-11]}$ Surgery can also be considered for the cases where conservative methods do not succeed. With surgery, the PM is relaxed, and the fibrous bands are excised to reduce the pressure on the sciatic nerve. ${ }^{[8,11,13,19]}$ We suggested medication (myorelaxants and nonsteroid anti-inflammatory drugs, vitamin $\mathrm{B}_{1}$ ) combined with physical therapy modalities (hotpack, electrotherapy, etc.) and exercises (especially stretching) to the patients diagnosed with PMS. 
In the literature, the use of UE in musculoskeletal disorders is rapidly increasing. It gives an opportunity for functional evaluation when the diagnosis is difficult and the clinic is incompatible with radiology. It has been found in literature that there are some UE studies in the areas of rotator cuff muscles, ${ }^{[12,32]}$ chronic neck pain, ${ }^{[5,33]}$ muscular dystrophy, ${ }^{[34]}$ athletes, ${ }^{[6,35]}$ chronic low back pain, ${ }^{[36,37]}$ and torticollis, etc. ${ }^{[38]}$ In our study, for the first time in the literature, we analyzed the UE characteristics of gluteal region muscles in PMS, and still no gold standard diagnostic method is obtained yet. In accordance with the literature, we found high UESR values and high elasticity on the affected side. The high strain ratio indicated the increase in tissue elasticity and hardening of the tissue. We also found that both PM and GM were structurally effected together with age, gender, weight, and height differences in PMS.

Care must be taken to position the patient. He should be lined in prone position. A few measurements should be taken consecutively for a correct measurement. The radiologist should gain experience before. As in other US studies, the experience and practice of the radiologist may affect the result of the study, so standardized methods are needed. Since only one radiologist was able to measure, only intraobserver measurements could be made and compliance was high. Interobserver variation of such a method will be the subject of future, large-sampled studies.

\section{Study limitations}

- The study included relatively small number of cases

- The control and the case groups were the same

- The painless side was accepted as normal

- UE standards have not been established yet in the literature

- There is need of new similar studies, to say more accurate information.

\section{Conclusion}

PMS is relatively less known and underestimated because it is confused with other clinical pathological conditions. Delays in its diagnosis may lead to chronic somatic dysfunction and muscle weakness. We showed that the muscle elasticity and hardness increased on the effected muscles in UE, which is an easy and cost-effective method. UE may provide early diagnosis of PMS and, consequently, a possibility of treatment with less invasive methods.

\section{Financial support and sponsorship}

Nil.

\section{Conflicts of interest}

There are no conflicts of interest.

\section{References}

1. Benson ER, Schutzer SF. Posttraumatic piriformis syndrome: Diagnosis and results of operative treatment. J Bone Joint Surg Am 1999;81:941-9.

2. Hopayian K, Danielyan A. Four symptoms define the piriformis syndrome: An updated systematic review of its clinical features. Eur J Orthop Surg Traumatol 2018;28:155-64.

3. Rossi P, Cardinali P, Serrao M, Parisi L, Bianco F, De Bac S, et al. Magnetic resonance imaging findings in piriformis syndrome: A case report. Arch Phys Med Rehabil 2001;82:519-21.

4. Brandenburg JE, Eby SF, Song P, Zhao H, Brault JS, Chen S, et al. Ultrasound elastography: The new frontier in direct measurement of muscle stiffness. Arch Phys Med Rehabil 2014;95:2207-19.

5. Grabowski PJ, Slane LC, Thelen DG, Obermire T, Lee KS. Evidence of generalized muscle stiffness in the presence of latent trigger points within infraspinatus. Arch Phys Med Rehabil 2018. pii: S0003-9993(18)30245-4.

6. Akkoc O, Caliskan E, Bayramoglu Z. Effects of passive muscle stiffness measured by shear wave elastography, muscle thickness, and body mass index on athletic performance in adolescent female basketball players. Med Ultrason 2018;20:170-6.

7. Fishman LM, Konnoth C, Rozner B. Botulinum neurotoxin type $\mathrm{B}$ and physical therapy in the treatment of piriformis syndrome: A dose-finding study. Am J Phys Med Rehabil 2004;83:42-50.

8. Boyajian-O'Neill LA, McClain RL, Coleman MK, Thomas PP. Diagnosis and management of piriformis syndrome: An osteopathic approach. J Am Osteopath Assoc 2008;108:657-64.

9. Freiberg AH, Vinke TH. Sciatica and the sacro-iliac joint. J Bone Joint Surg 1934;16:126-36.

10. Yoon SJ, Ho J, Kang HY, Lee SH, Kim KI, Shin WG, et al. Low-dose botulinum toxin type A for the treatment of refractory piriformis syndrome. Pharmacotherapy 2007;27:657-65.

11. Pace JB, Nagle D. Piriform syndrome. West J Med 1976;124:435-9.

12. Demirel A, Baykara M, Koca TT, Berk E. Ultrasound elastography-based assessment of the elasticity of the supraspinatus muscle in impingement syndrome: Does elastography has any diagnostic value? Clin Rheumatol 2018;37:1661-5.

13. Payne JM. Ultrasound-guided hip procedures. Phys Med Rehabil Clin N Am 2016;27:607-29.

14. Sharma T, Singla RK, Lalit M. Bilateral eventration of sciatic nerve. JNMA J Nepal Med Assoc 2010;50:309-12.

15. Jengojan S, Schellen C, Bodner G, Kasprian G. Imaging of the lumbosacral plexus: Diagnostics and treatment planning with high-resolution procedures. Radiologe 2017;57:195-203.

16. Papadopoulos EC, Khan SN. Piriformis syndrome and low back pain: A new classification and review of the literature. Orthop Clin North Am 2004;35:65-71.

17. Battaglia PJ, D'Angelo K, Kettner NW. Posterior, lateral, and anterior hip pain due to musculoskeletal origin: A narrative literature review of history, physical examination, and diagnostic imaging. J Chiropr Med 2016;15:281-93.

18. Tibor LM, Sekiya JK. Differential diagnosis of pain around the hip joint. Arthroscopy 2008;24:1407-21.

19. Benzon HT, Katz JA, Benzon HA, Iqbal MS. Piriformis syndrome: Anatomic considerations, a new injection technique, and a review of the literature. Anesthesiology 2003;98:1442-8.

20. Broadhurst NA, Simmons DN, Bond MJ. Piriformis syndrome: Correlation of muscle morphology with symptoms and signs. Arch Phys Med Rehabil 2004;85:2036-9.

21. Fanucci E, Masala S, Squillaci E, Sodani G, Varrucciu V, Ursone A, et al. Pyriformis muscle syndrome: CT/MR findings 
in the percutaneous therapy with botulinic toxin. Radiol Med 2003;105:69-75.

22. Ro TH, Edmonds L. Diagnosis and management of piriformis syndrome: A rare anatomic variant analyzed by magnetic resonance imaging. J Clin Imaging Sci 2018;8:6.

23. Bartret AL, Beaulieu CF, Lutz AM. Is it painful to be different? Sciatic nerve anatomical variants on MRI and their relationship to piriformis syndrome. Eur Radiol 2018; doi: 10.1007/ s00330-018-5447-6. [Epub ahead of print].

24. Huang ZF, Yang DS, Shi ZJ, Xiao J. Pathogenesis of piriformis syndrome: A magnetic resonance imaging-based comparison study. Zhonghua Yi Xue Za Zhi 2018;98:42-5.

25. Papadopoulos SM, McGillicuddy JE, Albers JW. Unusual cause of 'piriformis muscle syndrome'. Arch Neurol 1990;47:1144-6.

26. Hou SW, Merkle AN, Babb JS, McCabe R, Gyftopoulos S, Adler RS, et al. Shear wave ultrasound elastographic evaluation of the rotator cuff tendon. J Ultrasound Med 2017;36:95-106.

27. Krepkin K, Bruno M, Raya JG, Adler RS, Gyftopoulos S. Quantitative assessment of the supraspinatus tendon on MRI using T2/T2* mapping and shear-wave ultrasound elastography: A pilot study. Skeletal Radiol 2017;46:191-9.

28. Kosukegawa I, Yoshimoto M, Isogai S, Nonaka S, Yamashita T. Piriformis syndrome resulting from a rare anatomic variation. Spine (Phila Pa 1976). 2006 Aug 15;31(18):E664-6.

29. Parziale JR, Hudgins TH, Fishman LM. The piriformis syndrome. Am J Orthop (Belle Mead NJ). 1996 Dec; 25(12):819-23.

30. Hopayian K, Song F, Riera R, Sambandan S. The clinical features of the piriformis syndrome: A systematic review. Eur Spine J
2010;19:2095-109.

31. Fishman LM, Dombi GW, Michaelsen C, Ringel S, Rozbruch J, Rosner B, et al. Piriformis syndrome: Diagnosis, treatment, and outcome-A 10-year study. Arch Phys Med Rehabil 2002;83:295-301.

32. Kim K, Hwang HJ, Kim SG, Lee JH, Jeong WK. Can shoulder muscle activity be evaluated with ultrasound shear wave elastography? Clin Orthop Relat Res 2018;476:1276-83.

33. Ewertsen C, Carlsen J, Perveez MA, Schytz H. Reference values for shear wave elastography of neck and shoulder muscles in healthy individuals. Ultrasound Int Open 2018;4:E23-9.

34. Pichiecchio A, Alessandrino F, Bortolotto C, Cerica A, Rosti C, Raciti MV, et al. Muscle ultrasound elastography and MRI in preschool children with duchenne muscular dystrophy. Neuromuscul Disord 2018;28:476-83.

35. Inami T, Nakagawa K, Yonezu T, Fukano M, Higashihara A, Iizuka $\mathrm{S}$, et al. Tracking of time-dependent changes in muscle hardness after a full marathon. J Strength Cond Res 2018; [Epub Ahead of Print]. [doi: 10.1519/JSC.0000000000002495].

36. Creze M, Soubeyrand M, Yue JL, Gagey O, Maître X, Bellin MF, et al. Magnetic resonance elastography of the lumbar back muscles: A preliminary study. Clin Anat 2018;31:514-20.

37. KoppenhaverS, Kniss J, Lilley D, Oates M, Fernández-de-Las-PeñasC, Maher R, et al. Reliability of ultrasound shear-wave elastography in assessing low back musculature elasticity in asymptomatic individuals. J Electromyogr Kinesiol 2018;39:49-57.

38. Park GY, Kwon DR, Kwon DG. Shear wave sonoelastography in infants with congenital muscular torticollis. Medicine (Baltimore) 2018;97:e9818. 